\title{
Dexamethasone induces aberrant macrophage immune function and apoptosis
}

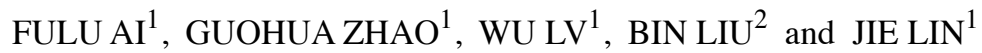 \\ ${ }^{1}$ Department of General Surgery (VIP ward), Cancer Hospital of China Medical University, Liaoning Cancer Hospital \\ and Institute; ${ }^{2}$ Department of Medical Oncology, Cancer Hospital of China Medical University, \\ Liaoning Cancer Hospital and Institute, Shenyang, Liaoning 110042, P.R. China
}

Received June 14, 2019; Accepted October 22, 2019

DOI: $10.3892 /$ or.2019.7434

\begin{abstract}
Glucocorticoids (GCs) are known potent clinical drugs, however, their mode of action is still complex and debatable. Macrophages are the most important target of GCs and play a key role in tumor immunity in vivo, but their relationship is also controversial. In the present study, the lentivirus system was used to overexpress and knock down the level of transcription factor Krüppel-like factor 9 (KLF9). The results revealed that dexamethasone (Dex) induced ROS generation and mitochondria-dependent apoptosis in RAW 264.7 cells via the KLF9. In addition, overexpression of KLF9 significantly increased apoptosis of RAW 264.7 cells. Notably, ELISA assay revealed that increased expression of KLF9 inhibited LPS-induced $C O X-2$ expression and reduced COX-2-derived prostaglandin E2 and pro-inflammatory cytokine secretion. Furthermore, a co-culture system was used to reveal that overexpression of KLF9 in RAW 264.7 cells promoted HepG 2 cell survival. In summary, it is reported that KLF9 promoted apoptosis of proinflammatory macrophages, and suppressed the antitumor effects, which can be selectively targeted by GCs as a novel mechanism to suppress antineoplastic activity.
\end{abstract}

\section{Introduction}

Glucocorticoids (GCs) are essential circadian steroid hormones that regulate perinatal development, memory, immune system function, and metabolism, as well as process emotional input (1) GCs are frequently used to treat rheumatoid arthritis due to their potent anti-inflammatory effect. The potent immunosuppressive effects of GCs are mediated by a series of transcriptional events, primarily by binding to the cytosolic

Correspondence to: Dr Jie Lin, Department of General Surgery (VIP ward), Cancer Hospital of China Medical University, Liaoning Cancer Hospital and Institute, 44 Xiaoheyan Road, Dadong, Shenyang, Liaoning 110042, P.R. China

E-mail: linjie@cmu.edu.cn

Key words: glucocorticoids, macrophages, dexamethasone, KLF9, apoptosis, immunosuppression, $\mathrm{COX}-2$ glucocorticoid receptor (GR). Translocated GR can directly bind to a canonical GC response element or act indirectly by interaction with other transcription factors (2). The generally accepted view of anti-inflammatory actions on macrophages is through the suppression of $\mathrm{NF}-\kappa \mathrm{B}$ activity, thus inhibiting the transcription of proinflammatory genes $(3,4)$. Accumulating evidence has demonstrated that other than the ability to suppress macrophage production of proinflammatory mediators, GCs can directly induce specific changes in cell survival, proliferation, and phagocytosis to suppress inflammation (5). Direct GC action on macrophages was suggested to suppress immunocompetence and promote antitumor gene transcription via GR activity. Synthetic glucocorticoids, such as dexamethasone (Dex), display potent inflammatory suppressive properties and are used to treat various inflammatory and autoimmune conditions $(2,6)$.

Macrophages are resident phagocytic cells found in lymphoid and non-lymphoid tissues. Macrophages are critical effectors involved in steady-state tissue homeostasis, via the clearance of apoptotic cells, production of growth factors, and regulation of inflammation and the innate immune response. Macrophages are the first line of defense against microorganisms, which is accomplished by phagocytosis and the production of inflammatory cytokines. In addition, inflammatory chemoattractants, induced by distant primary tumors, influence the attraction of macrophages in secondary sites before metastasis. The presence of macrophages within tumors indicates a poor prognosis, as they enhance angiogenesis and metastases. However, the migration of activated macrophages has not yet been elucidated.

Krüppel-like factor 9 (KLF9), also called basic transcription element-binding protein-1 (Bteb1), is a ubiquitously expressed member of the $\mathrm{C} 2 \mathrm{H} 2$-type zinc finger family (7). A recent study suggested that KLF9 plays an important role in regulating animal development and differentiation of various cell types (8). KLF9 can be induced by several physiological or pathological stresses. Current experimental evidence indicates that KLF9 plays a key hormone-dependent role in liver gluconeogenesis (9). Notably, a recent study indicated that KLF9 is induced by NF-E2-like basic leucine zipper transcriptional activator (Nrf2), thereby promoting cell oxidative stress (7).

Cyclooxygenase-2 (COX-2) levels are increased in tumors by several proinflammatory cytokines, which are secreted by 
macrophages, lymphocytes, and even epithelial cells $(10,11)$. Increased COX-2 levels have been reported in many malignant gastrointestinal tumors, including cholangiocarcinoma. Inhibition of COX-2 may suppress the development of epithelial cell malignancies in the gastrointestinal tract (12-14). In addition, COX-2 has been revealed to modulate the fate of colon cancer cell lines by generating prostanoids (15-17). These observations indicate that $C O X-2$ expression and prostanoid generation may play a fundamental role in the initiation and promotion of cancers arising from inflamed tissues, such as cholangiocarcinoma (18). However, it is unknown whether COX-2 can modulate apoptosis of immune cells, such as macrophages.

In the present study, the inhibitory effects and underlying molecular mechanisms of GCs on the inflammatory response were further investigated in lipopolysaccharide (LPS)-stimulated macrophages. Results indicated that KLF9 significantly suppressed LPS-induced intracellular reactive oxygen species (ROS) production and COX-2, while promoting the production of inflammatory factors in macrophages.

\section{Materials and methods}

Cell culture. RAW 264.7, murine macrophage cell line (TIB-71) and HepG2, liver cancer cell line (HB-8065) were purchased from the ATCC and were cultured in DMEM with 10\% FBS purchased from Gibco; Thermo Fisher Scientific, Inc. and 1\% penicillin-streptomycin in a humidified $5 \% \mathrm{CO}_{2}$ incubator at $37^{\circ} \mathrm{C}$. During maintenance, the cells were sub-cultured every 3 or 4 days.

Real-time quantitative PCR. Total RNA was extracted from RAW 254.7 cells using the TRIzol-based method (product no. 10296010; Invitrogen; Thermo Fisher Scientific, Inc.) and reverse-transcribed using Super-Script III reverse transcriptase (product no. 11752250; Invitrogen; Thermo Fisher Scientific, Inc.). The realtime quantitative-PCR was performed using the SYBR Green PCR Master mix (product no. A6001; Promega Corp.). The real-time quantitative-PCR conditions were $95^{\circ} \mathrm{C}$ for $30 \mathrm{sec}$, followed by 30 cycles at $95^{\circ} \mathrm{C}$ for $30 \mathrm{sec}$, $57^{\circ} \mathrm{C}$ for $1 \mathrm{~min}$, and $72^{\circ} \mathrm{C}$ for $30 \mathrm{sec}$. All quantitative-PCR data were normalized to the level GAPDH. Specific primers used are listed in Table I.

Chromatin immunoprecipitation (ChIP) assay. The RAW 264.7 cells $\left(2 \times 10^{7}\right)$ were fixed with $1 \%$ formaldehyde at room temperature for $30 \mathrm{~min}$, then chromatin was extracted and the cells were sonicated to shear the chromatin and immunoprecipitated with 2-5 $\mu \mathrm{g}$ antibodies specific for GR (product no. SAB4501309; Sigma-Aldrich; Merck KGaA), KLF9 (product no. ab227920; Abcam) or non-specific IgG (product no. sc-2027; Santa Cruz Biotechnology, Inc.). Then, the immunoprecipitants were isolated using protein $\mathrm{G}$ agarose beads (product no. 15920010; Invitrogen; Thermo Fisher Scientific, Inc.), followed by extensive washing and elution with $2 \%$ SDS in $0.5 \mathrm{M} \mathrm{NaHCO}$. After reversing the cross-links, the input DNA and immunoprecipitated DNA fragments were quantified by qPCR in both the input and precipitated samples. ChIP primer sequences are listed in Table II.
Western blot analysis. Whole-cell extracts were extracted from cultured RAW 264.7 cells in lysis buffer $(20 \mathrm{mM}$ Tris-Cl pH 7.5, $140 \mathrm{mM} \mathrm{NaCl}, 1 \mathrm{mM} \mathrm{CaCl}$ and $\mathrm{MgCl}_{2}$, $10 \mathrm{mM} \mathrm{NaF}, 1 \%$ NP-40, $10 \%$ glycerol, $2 \mathrm{mM}$ Na-Vanadate, and $1 \mathrm{mM}$ PMSF) supplemented with protease inhibitor cocktail (Roche Diagnostics). Homogenates were sonicated and centrifuged at $4^{\circ} \mathrm{C}$ for $15 \mathrm{~min}$, and the supernatants were used for western blotting. Protein concentrations of the samples in the experiments were determined by BCA (Bio-Rad protein assay kit). Proteins $(20-50 \mu \mathrm{g})$ were subjected to $10 \%$ SDS-polyacrylamide gels. Proteins were separated by SDS-PAGE and transferred to a PVDF membrane (EMD Millipore). After being blocked with 5\% non-fat milk, the membranes were incubated with the antibodies overnight at $4^{\circ} \mathrm{C}$. Immunoblotting was performed using the following primary antibodies: KLF9 (1:1,000; product no. A7196; ABclonal), COX-2 (1:1,000; product no. A1253; ABclonal), $\beta$-tubulin (1:1,000; product no. AC010; ABclonal), caspase-9 (1:500; product no. ab52298; Abcam), cleaved caspase-9 (1:500; product no. ab2324, Abcam), caspase-3 (1:500; product no. ab13847; Abcam), cleaved caspase-3 (1:500; product no. ab2302, Abcam), cytochrome $c$ (Cyt- $c$ ) (1:500; product no. A0225; ABclonal). Then the PVDF membranes were incubated with the secondary antibodies for $1 \mathrm{~h}$ at room temperature. HRP-conjugated secondary antibody (1:5,000; product no. sc-2357 and sc-2005; Santa Cruz Biotechnology, Inc.) was used according to the manufacturer. ECL reagent was used for visualization (Genestar). Western blots were quantified by densitometry using ImageJ (1.52q for Windows; National Institutes of Health).

ELISA assays. RAW 264.7 cells $\left(1 \times 10^{6}\right.$ cells/well) were transfected with Lenti-KLF9 for $48 \mathrm{~h}$ in 6-well plates, then LPS $(1 \mu \mathrm{g} / \mathrm{ml})$ was added to the cultured wells for another $24 \mathrm{~h}$. The cultured supernatant was collected and the levels of PGE2, IL-1 $\beta$, IL-6, and TNF- $\alpha$ were assessed using ELISA assays. ELISA kits were purchased from R\&D Systems for IL- $1 \beta$, IL-6 and TNF- $\alpha$ (product no. A54609) and PGE2 (product no. A50432) was purchased from EpiGentek.

Transient transfection and luciferase reporter assays. RAW264.7 cells were transiently co-transfected with KLF9-Luc or COX-2-Luc and the respective expressed plasmid ( $G R$ or KLF9) using Lipofectamine 2000 (product no. 11668019; Invitrogen; Thermo Fisher Scientific, Inc.). After $48 \mathrm{~h}$, the cells were treated with Dex or LPS for another $3 \mathrm{~h}$. Then, reporter gene assays were performed using a luciferase assay system (product no. E2810; Promega Corp.). The internal reference was determined by measuring the TK (mammalian vector for weak constitutive expression of humanized Renilla luciferase) bioluminescence intensity.

TUNEL assay. A TUNEL assay was performed to detect the apoptotic RAW 264.7 cells. The TUNEL assay was performed using the In Situ Cell Death Detection kit (Roche Diagnostics). The images for assessing the apoptotic cells were obtained by fluorescence microscope (ZEISS AG). TUNEL-positive cells in different groups were calculated by randomly selecting six different fields and at least six data of each group were used for analysis. ImageJ was used to count the percentage of apoptotic 
Table I. The sequences of the primers used for RT-qPCR.

\begin{tabular}{ll}
\hline Primer name & \multicolumn{1}{c}{ Sequence (5'-3') } \\
\hline KLF9 & F: 5'-CGAGCGGCTGCGACTACCTG-3' \\
& R: 5'-GGGCTGTGGGAAGGACTCGAC-3' \\
COX-2 & F: 5'-CATCCCCTTCCTGCGAAGTT-3' \\
& R: 5'-GGCCCTGGTGTAGTAGGAGA-3' \\
IL-6 & F: 5'-CCACGGCCTTCCCTACTTC-3' \\
& R: 5'-TTGGGAGTGGTATCCTCTGTGA-3' \\
IL-1 $\beta$ & F: 5'-AGTTGACGGACCCCAAAAGAT-3' \\
& R: 5'-GGACAGCCCAGGTCAAAGG-3' \\
TNF- $\alpha$ & F: 5'-CACCGTCAGCCGATTTGC-3' \\
& R: 5'-TTGACGGCAGAGAGGAGGTT-3' \\
GAPDH & F: 5'-CAAGGCTGTGGGCAAGGT-3' \\
& R: 5'-GGAAGGCCATGCCAGTGA-3'
\end{tabular}

KLF9, Krüppel-like factor 9; COX-2, cyclooxygenase-2; F, forward; $\mathrm{R}$, reverse.

Table II. The sequences of the primers used for ChIP-qPCR.

\begin{tabular}{lc}
\hline Primer name & \multicolumn{1}{c}{ Sequence (5'-3') } \\
\hline KLF9 ChIP & F: 5'-AGAGGCGCGGCGCGGCAGG-3' \\
& R: 5'-GTCGGAGTCCCAGAGAAAC-3' \\
COX-2 ChIP & F: 5'-CCACTACGTCACGTGGAGT-3' \\
& R: 5'-GCTGAGTTCCTTCGTGAGCA-3' \\
\hline
\end{tabular}

KLF9, Krüppel-like factor 9; COX-2, cyclooxygenase-2; F, forward; $\mathrm{R}$, reverse.

cells subjected for statistical analysis. The nucleus was counterstained with DAPI fluorescence dye (product no. c1005; Beyotime Institute of Biotechnology) for total cell count.

Cell co-culture system. Transwell plates (product no. 3378; Corning, Inc.) were used to construct a co-culture system. In brief, serum-free single cell suspensions $\left(3 \times 10^{4}\right.$ cells $\left./ \mathrm{ml}\right)$ were prepared. The upper Transwell chamber was filled with $100 \mu \mathrm{l}$ HepG 2 cells, while RAW 264.7 cells with RPMI-1640 medium (20\% FBS) was added into the lower chamber. Cells were cultured in a humidified $5 \% \mathrm{CO}_{2}$ incubator at $37^{\circ} \mathrm{C}$. During maintenance, the cell proliferation assay was performed once a day for 5 consecutive days.

Cell proliferation assay. A Cell Counting Kit- 8 (product no. ab228554; Abcam) was used to assess cell proliferation. Cells were cultured at $37^{\circ} \mathrm{C}$ in a $5 \% \mathrm{CO}_{2}$ incubator. Subsequently, $10 \mu \mathrm{l}$ CCK-8 reagent was added into each well and the plate was incubated for 1-4 h. OD values at $450 \mathrm{~nm}$ were assessed for cell proliferation.

Statistical analyses. The quantitative data are represented as the mean \pm SD of at least three independent experiments. Two-tailed Student's t-test was used to compare the differences between 2 groups. One-way ANOVA with Bonferroni correction was used for multiple comparisons. Statistical significance was defined as $\mathrm{P}<0.05\left({ }^{*} \mathrm{P}<0.05\right.$; ${ }^{* *} \mathrm{P}<0.01 ;{ }^{* * *} \mathrm{P}<0.005 ;{ }^{* * * *} \mathrm{P}<0.001$, as indicated in the figures and legends). Analysis was performed using GraphPad Prism (GraphPad Software, Inc.).

\section{Results}

Dex induces KLF9 expression in macrophage cells through $G R$. The molecular mechanism of GCs which promote macrophages apoptosis and tumor growth remains largely unclear. In order to investigate the regulated effect of GCs, the expression level of $K L F 9$, which plays a key role in mitochondria-dependent cell death, was first examined in macrophages after Dex treatment. The results revealed that Dex significantly induced the expression of KLF9 in RAW 264.7 cells (Fig. 1A and B). As is recognized, the transcriptional regulation of the function of GCs is largely due to GR, an important hormone-sensitive transcription factor of the nuclear receptor superfamily. To further investigate the regulated function of Dex, promoter activity analysis of $K L F 9$ was performed. The -1371 to +226 region of $K L F 9$ promoter which was fused to the luciferase reporter gene system (KLF9-Luc) was constructed. Then, $G R$ expression plasmid or control plasmid was co-transfected with KLF9-Luc into RAW 264.7 cells. The results revealed that GR caused significant activation of the promoter with or without Dex (Fig. 1C). Subsequently, a potential GR response element site in the $K L F 9$ promoter that mediated the stimulatory effect of Dex/GR was revealed using the promoter deletion and mutation assays. ChIP assays confirmed that the GR was recruited to the KLF9 promoter region and mediated gene expression after Dex treatment (Fig. 1D). In another experiment, it was revealed that RU486, a GR antagonist, almost completely abolished the Dex/GR-mediated increased KLF9 level in RAW 264.7 cells (Fig. 1E and F). These results indicated that KLF9 was a GC- regulated gene in macrophage cells, and it was directly regulated by GR.

Overexpression of KLF9 induces apoptosis in macrophage cells. GCs were previously demonstrated to activate the intrinsic apoptotic pathways in a tumor model (4). in the present study, it was proposed that KLF9, which was induced by Dex, would activate apoptosis in macrophage cells. To investigate whether overexpression of $K L F 9$ would induce the apoptosis of macrophages, the $K L F 9$ lentivirus-expressing system (Lenti-KLF9) was generated and infected RAW 264.7 cells. The mRNA and protein level of KLF9 were dramatically elevated after Lenti-KLF9 transfected (Fig. 2A and B). The proportion of viable and apoptotic cells were analyzed by Trypan blue exclusion experiment and TUNEL analysis $48 \mathrm{~h}$ after Lenti-KLF9 transfection. The proportion of viable cells was significantly decreased in the $K L F 9$ overexpression group compared to the control group (Fig. 2C). Consistent with the decreased number of viable cells, the number of apoptotic cells was increased (Fig. 2D). It was therefore determined whether the decrease in cell viability could be attributed to the induction of apoptosis. Subsequently, the analysis of mitochondrial generated ROS was performed by a MitoSOX staining experiment to determine whether the mitochondrial-mediated 
A

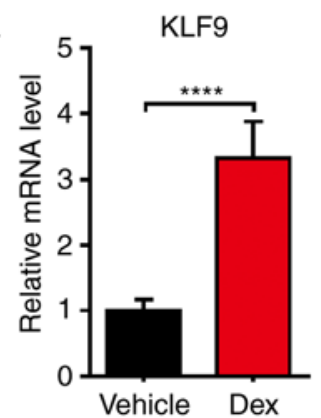

C

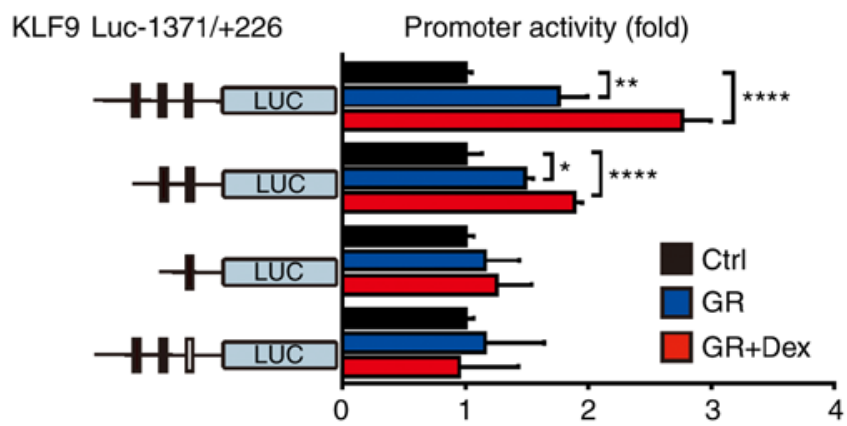

B

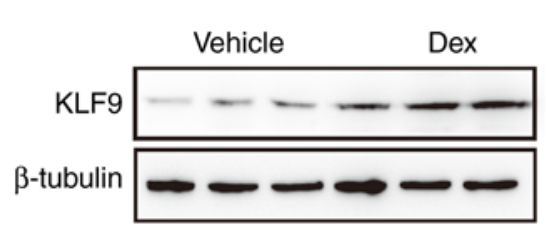

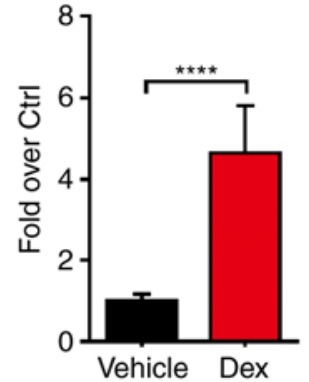

Promoter of KLF9
D

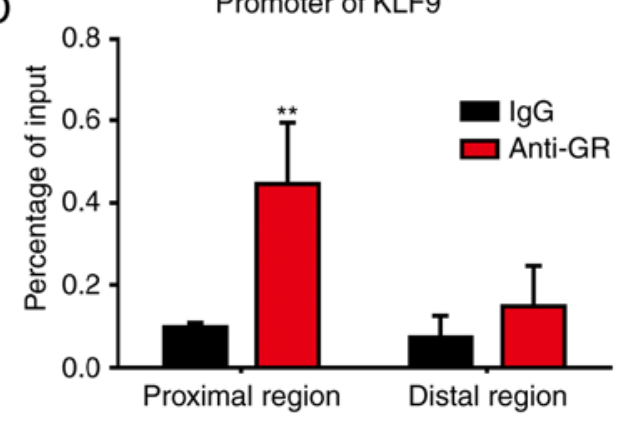

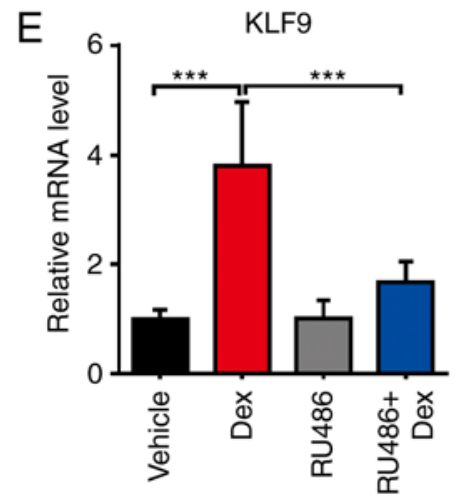
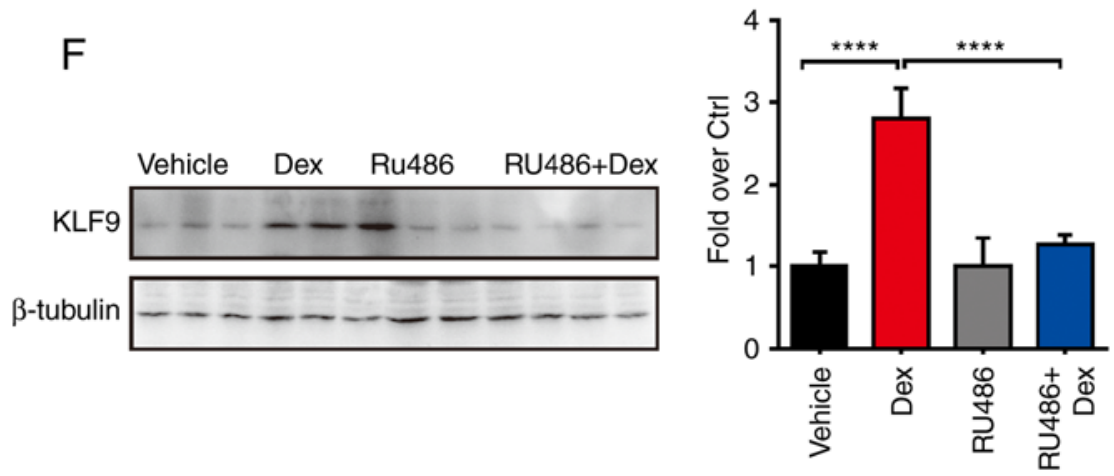

Figure 1. Expression of KLF9 is increased by Dex in RAW264.7 cells. (A) Real-time quantitative PCR analysis of the mRNA level of KLF9 in RAW264.7 cells $16 \mathrm{~h}$ after treatment with saline or Dex $(100 \mathrm{nM})(\mathrm{n}=5)$. (B) Western blot analysis of KLF9 in RAW264.7 cells as described in A; quantitative data are on the right. (C) A series of promoter activity of KLF9 was detected in RAW264.7 cells with saline or Dex (100 nM) treatment. (D) A ChIP assay was performed using anti-GR antibody in RAW264.7 cells. (E) Real-time quantitative PCR analysis of KLF9 in RAW264.7 cells treated with $100 \mathrm{nM}$ Dex or with $10 \mu$ M of the GR antagonist RU486 for $16 \mathrm{~h}$. (F) Western blot analysis of the protein level of KLF9 described in E; quantitative data are on the right. Experiments were performed in triplicate. Error bars represent the standard deviation ${ }^{*} \mathrm{P}<0.05 ;{ }^{* *} \mathrm{P}<0.01 ;{ }^{* * * *} \mathrm{P}<0.005 ;{ }^{* * * * *} \mathrm{P}<0.001$. KLF9, Krüppel-like factor 9; Dex, dexamethasone; ChIP, chromatin immunoprecipitation; GR, glucocorticoid receptor.

apoptosis pathway was involved. The results revealed that cells in the KLF9 group exhibited significantly more positive ROS generation than the control group (Fig. 2E). To further examine the mitochondrial effect induced by KLF9, the proteins of the mitochondrial apoptosis pathway in macrophage cells were next assessed. Western blot analysis revealed that the apoptosis-related protein levels of procaspase-9, cleaved caspase- 9 , procaspase-3, cleaved caspase- 3 and Cyt- $c$ of the KLF9 group were increased compared to the control group (Fig. 2F). Collectively, it was concluded that KLF9 may play an important role in the macrophage cells apoptosis pathway by promoting mitochondrial ROS generation and Cyt $c$ release.

KLF9 knockdown protects against dexamethasone-induced apoptosis in macrophage cells. It was previously revealed that KLF9 mediated the signaling pathway of Dex-induced mitochondrial injury and macrophage cells apoptosis. Therefore, it was hypothesized whether decreased $K L F 9$ expression level would inactivate the intrinsic apoptotic pathways induced by Dex. The sh $K L F 9$ lentivirus-knockdown system (Lenti-shKLF9) was generated and it was revealed that the mRNA and protein level of $K L F 9$ was suppressed by Lenti-shKLF9 with Dex treatment (Fig. $3 \mathrm{~A}$ and B). Initially, as assessed by the Trypan blue, it was observed that RAW 264.7 cells which were treated with Dex and transfected with Lenti-sh KLF9 exhibited a significant increased survival rate compared to Dex treatment alone (Fig. 3C). In addition, TUNEL assay revealed that the number of apoptotic cells in the Dex treatment group was higher than in the Dex+shKLF9 treatment group, which was exposed to Dex and transfected with Lenti-shKLF9 for $72 \mathrm{~h}$ (Fig. 3D). These results indicated that suppressed KLF9 level could markedly inhibit the apoptosis of RAW 264.7 cells induced by Dex. The analysis 


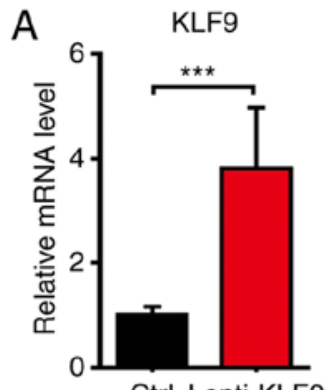

B
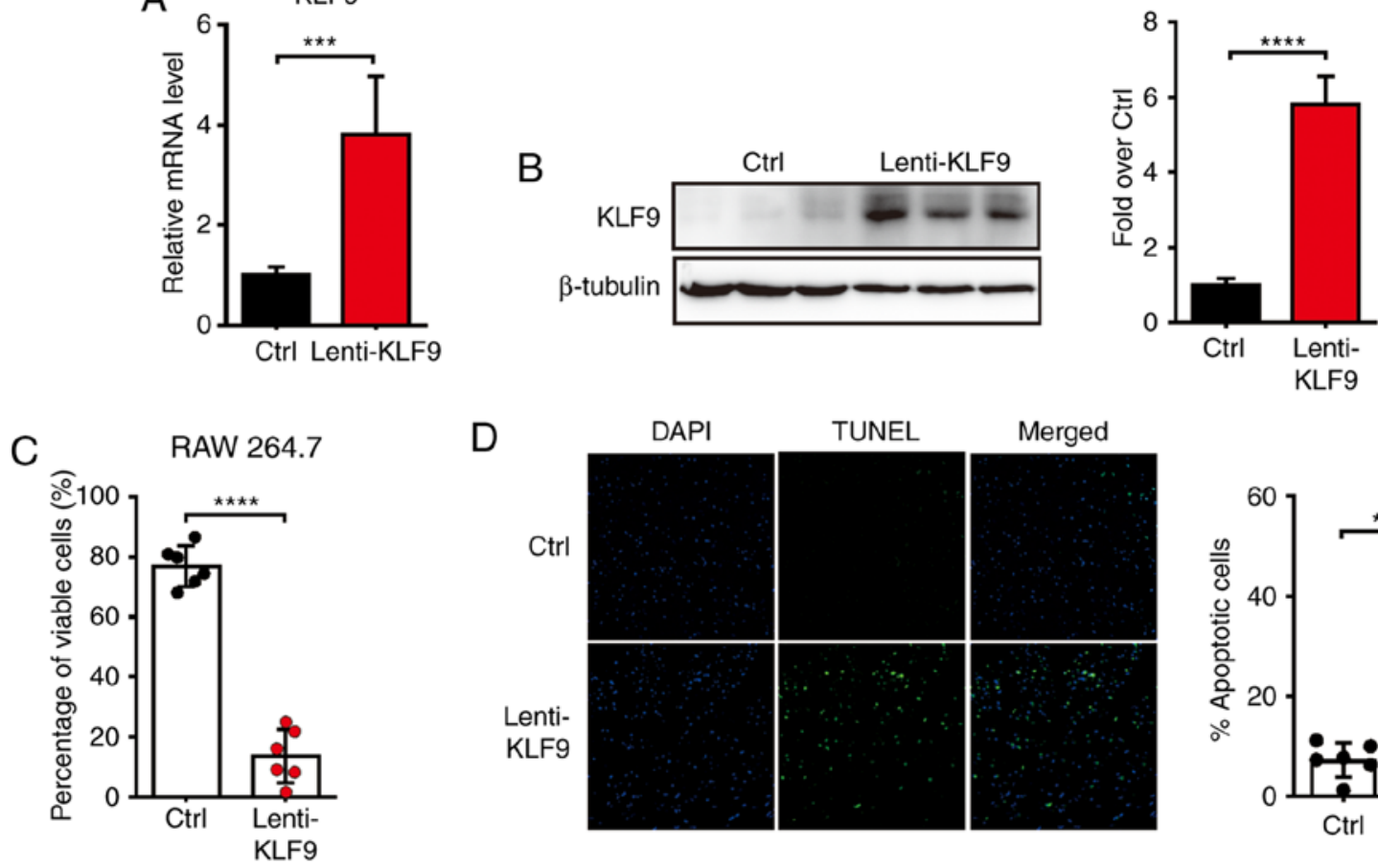

TUNEL
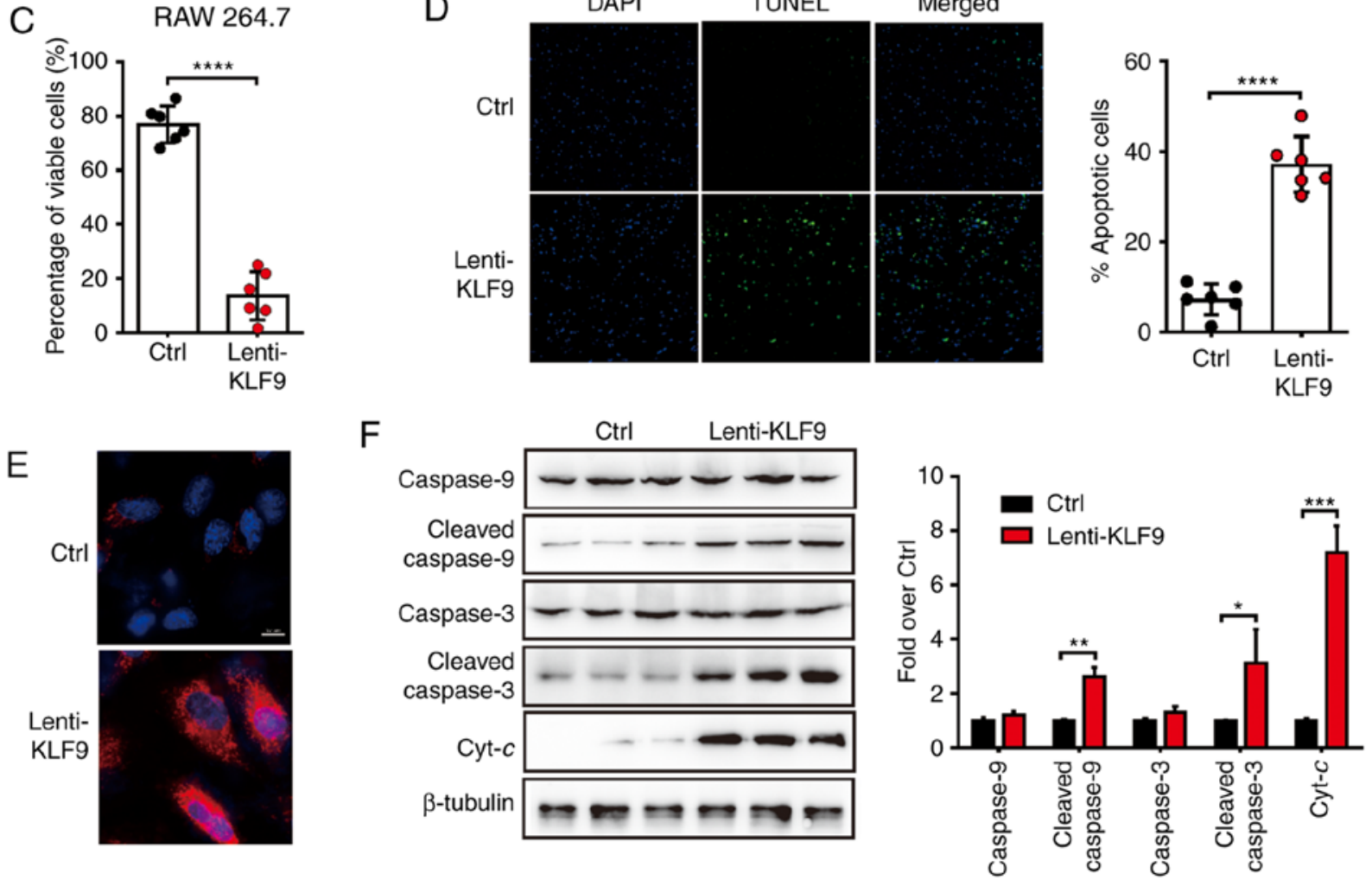

Figure 2. KLF9 increases apoptosis of RAW264.7 cells and activates the mitochondrial apoptotic pathway. (A) Real-time quantitative PCR analysis of the mRNA level of KLF9 in RAW264.7 cells $48 \mathrm{~h}$ after infection with control or KLF9 lentivirus (n=5). (B) Western blot analysis of the protein level of KLF9 in RAW264.7 cells as described in A; quantitative data are on the right. (C) Quantitative data of viable cells assessed by the Trypan blue exclusion method. (D) TUNEL assay revealed apoptosis of RAW264.7 cells in KLF9 and the control groups. Quantitative data of the percentage of TUNEL-positive cells is presented on the right. (E) Mitochondrial ROS generation in RAW264.7 cells were analyzed by immunofluorescence (red). Nuclei were stained with DAPI (blue). (F) Western blot analysis was performed for the detection of the mitochondrial-dependent apoptotic pathway including procaspase-9, cleaved caspase-9, procaspase-3, cleaved caspase-3 and Cyt- $c$ proteins, quantitative data are on the right. Experiments were performed in triplicate. Error bars represent the standard deviation $\left({ }^{*} \mathrm{P}<0.05 ;{ }^{* *} \mathrm{P}<0.01 ;{ }^{* * * *} \mathrm{P}<0.005 ;{ }^{* * * *} \mathrm{P}<0.001\right)$. KLF9, Krüppel-like factor 9; Dex, dexamethasone; ROS, reactive oxygen species; Cyt $-c$, cytochrome $c$.

of MitoSOX-positive staining revealed that RAW 264.7 cells in the Dex $+\operatorname{sh} K L F 9$ group exhibited significant less ROS generation than the Dex group (Fig. 3E). The apoptosis-related protein levels were next investigated and it was revealed that procaspase-9, cleaved caspase- 9 , procaspase-3, cleaved caspase- 3 and Cyt $-c$ protein levels of the Dex $+\operatorname{sh} K L F 9$ group were decreased compared to the Dex group (Fig. 3F). Collectively, it was concluded that Dex could influence the viability of macrophage cells by the KLF9-mediated mitochondrial apoptosis pathway.

KLF9 overexpression reduces the LPS-induced inflammatory cytokine release in $R A W 264.7$ cells. It was previously determined that KLF9 mediated macrophage apoptosis which was induced by Dex. Furthermore, it was then determined whether KLF9 influenced the function of macrophages. It is recognized that LPS can stimulate inflammatory cytokine production. Thus, the expressed and secreted level of inflammatory cytokines including IL- $1 \beta$, IL- 6 , and TNF- $\alpha$, which are potent antitumor factors released from macrophage cells, were assessed. The results revealed that LPS significantly increased the mRNA levels of these inflammatory cytokines, and KLF9 could decrease such variations (Fig. 4A). In addition, KLF9 also reduced LPS-induced secretion of these inflammatory cytokines (Fig. 4B). These results indicated that KLF9 could destroy the function of macrophages by reducing the inflammatory cytokine production and secretion. Subsequently, it was assessed whether $K L F 9$ overexpression in macrophages 
A

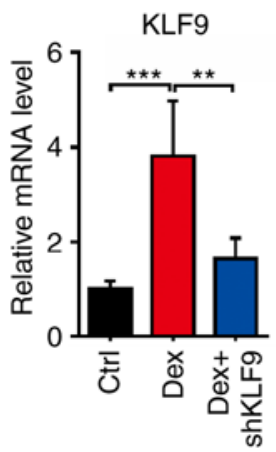

B

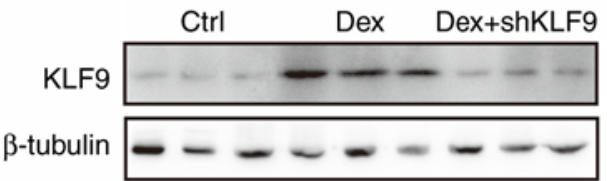

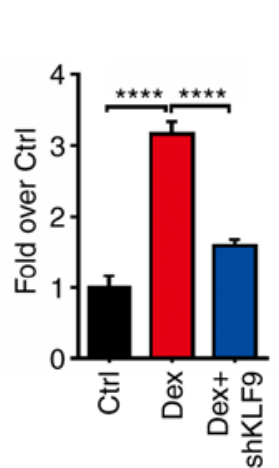

C

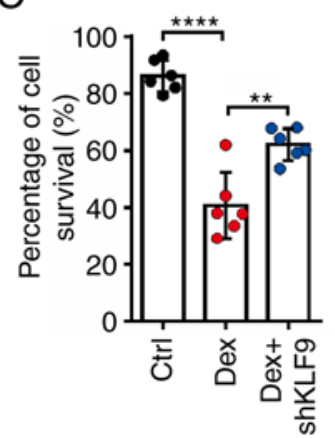

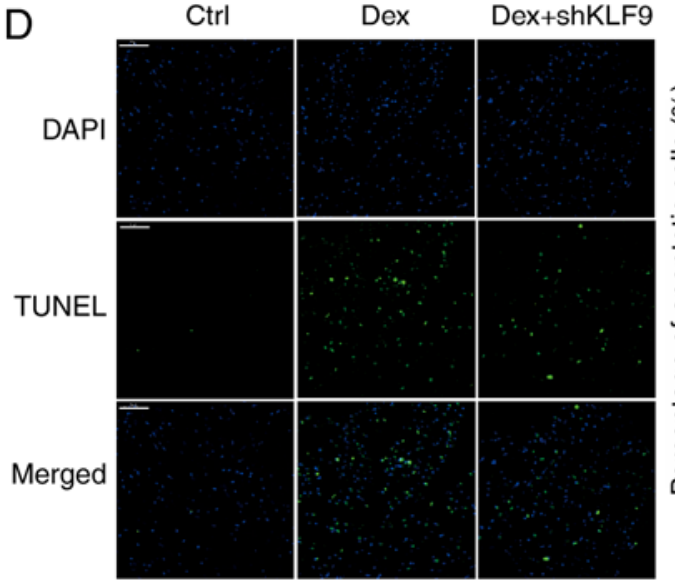

$\mathrm{F}$

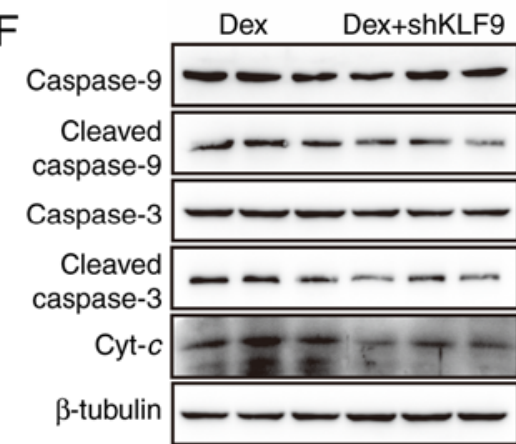

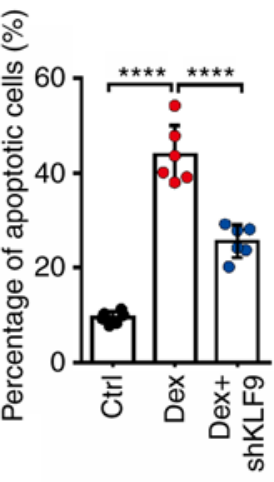

E

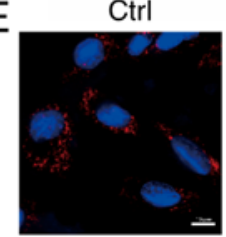

Dex+shKLF9

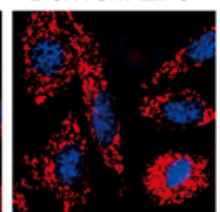

Figure 3. Knockdown of KLF9 decreases apoptosis and inhibits the activation of the mitochondrial apoptotic pathway induced by Dex in RAW264.7 cells. (A) Real-time quantitative PCR analysis of the mRNA level of KLF9 in RAW264.7 cells treated with Dex or saline after infection with control or KLF9 lentivirus for $48 \mathrm{~h}$. (B) Western blot analysis of the protein level of KLF9 in RAW264.7 cells as described in A. (C) Viable cells were analyzed using the Trypan blue exclusion method. (D) A TUNEL assay revealed the apoptosis of RAW264.7 cells in different groups. Quantitative data of TUNEL-positive cell percentages are on the right. (E) Mitochondrial ROS generation in RAW264.7 cells were analyzed by immunofluorescence. Nuclei were stained with DAPI (blue). (F) Western blot analysis was performed for the detection of the mitochondrial-dependent apoptotic pathway including procaspase-9, cleaved caspase-9, procaspase-3, cleaved caspase-3 and Cyt- $c$ proteins; quantitative data are on the right. Experiments were performed in triplicate. Error bars represent the standard deviation $\left({ }^{*} \mathrm{P}<0.05 ;{ }^{* *} \mathrm{P}<0.01 ;{ }^{* * *} \mathrm{P}<0.005 ;{ }^{* * * * *} \mathrm{P}<0.001\right)$. KLF9, Krüppel-like factor 9; Dex, dexamethasone; ROS, reactive oxygen species; Cyt-c, cytochrome $c$.

had an effect in HepG2 liver cancer cell proliferation using an in vitro co-culture system. The results demonstrated that the supernatant of macrophages stimulated with LPS significantly suppressed HepG2 liver cancer cell proliferation, however, this effect was alleviated following transfection with $K L F 9$ (Fig. 4C). Then, it was assessed whether KLF9 could regulate the level of PEG2, which plays a key role in mediating the microenvironment of liver cancer. Notably, it was revealed that KLF9 significantly suppressed LPS-induced PGE2 production in macrophage cells compared to the LPS group and there was a slight difference between the control and KLF9 group without LPS treatment (Fig. 4D).

KLF9 suppresses COX-2 levels. The response to LPS of KLF9-suppressed RAW 264.7 cells largely resided in the domain of transcriptional regulation. Thus, it was determined whether KLF9 directly regulated the expression of $C O X-2$. The mRNA and protein levels of $C O X-2$ were decreased by KLF9 compared to the control group as determined using the Real-time PCR and western blot assays in 
A

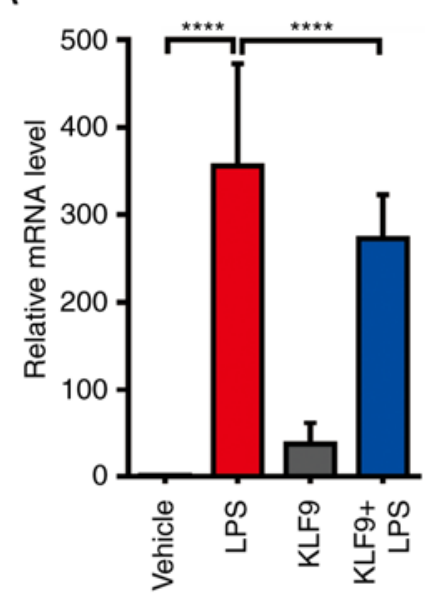

B

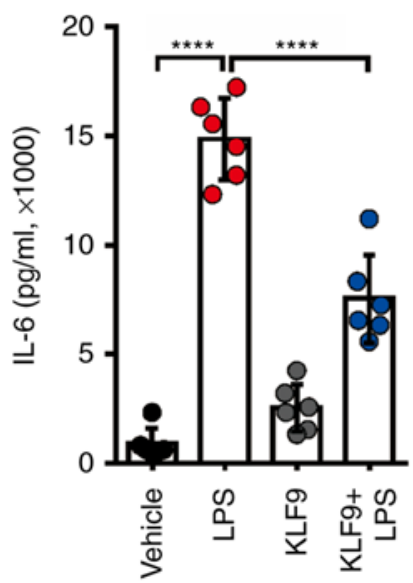

IL-1 $\beta$

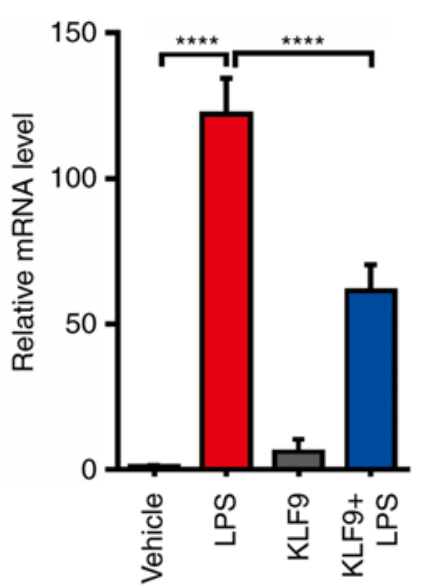

IL-1 $\beta$

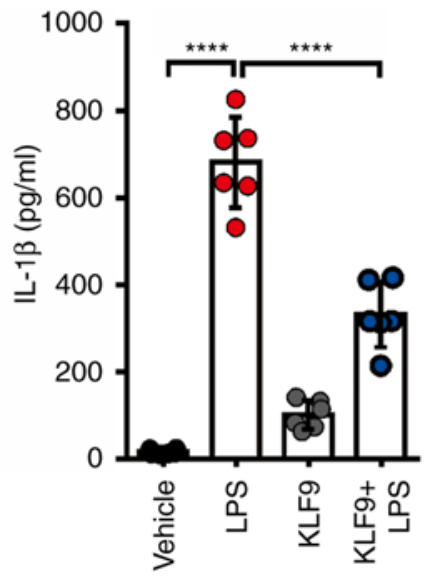

TNF- $\alpha$
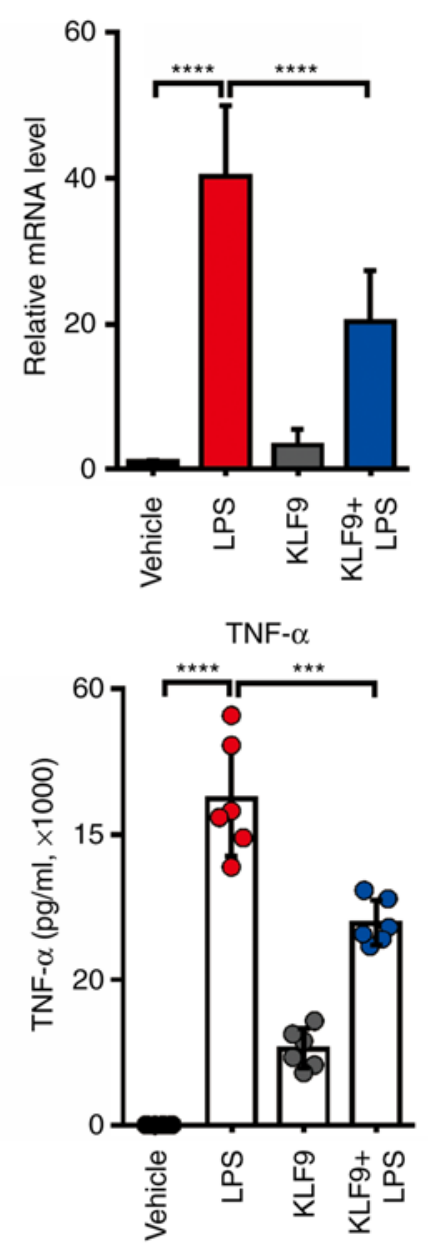

C

HepG2

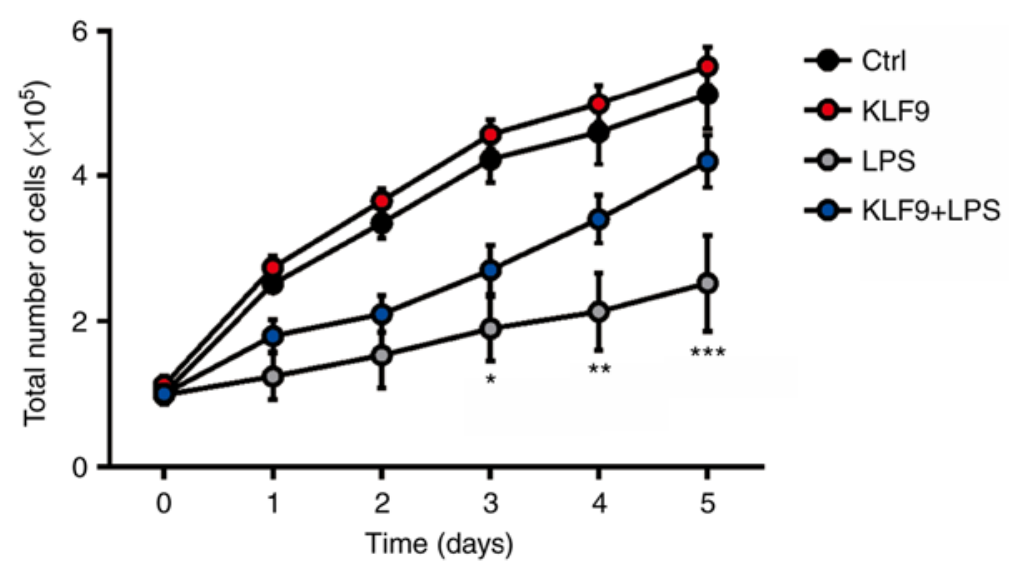

PGE2

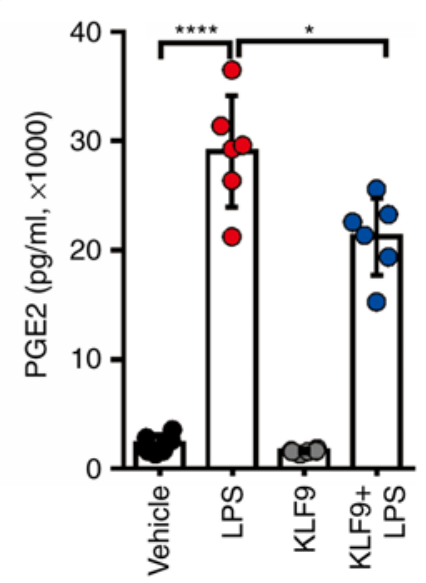

Figure 4. Effects of KLF9 on the production of proinflammatory cytokines from LPS-induced RAW264.7 cells. (A) RAW264.7 cells were transfected with Lenti-KLF9 and then co-treated with $1 \mu \mathrm{g} / \mathrm{ml}$ LPS for $24 \mathrm{~h}$. The mRNA levels of TNF- $\alpha$, IL-1 $\beta$, IL- 6 are analyzed by Real-time quantitative PCR analysis. (B) An ELISA assay was used to determine TNF- $\alpha$, IL-1 $\beta$, IL-6 production in the supernatants of RAW264.7 cells following treatment with $1 \mu$ g/ml LPS and transfected with Lenti-KLF9 or control, respectively. (C) Proliferation rate of HepG2 cells was assessed using CCK8 after co-culture with RAW264.7 cells following treatment with $1 \mu \mathrm{g} / \mathrm{ml}$ LPS and transfected with Lenti-KLF9 or control, respectively. (D) ELISA assay was used to determine PGE2 production in the supernatants of RAW264.7 cells following treatment with $1 \mu \mathrm{g} / \mathrm{ml}$ LPS and transfected with Lenti-KLF9 or control, respectively. Experiments were performed in triplicate. Error bars represent the standard deviation $\left({ }^{*} \mathrm{P}<0.05 ;{ }^{* *} \mathrm{P}<0.01 ;{ }^{* * *} \mathrm{P}<0.005 ;{ }^{* * * *} \mathrm{P}<0.001\right)$. KLF9, Krüppel-like factor 9 ; LPS, lipopolysaccharide.

RAW 264.7 cells (Fig. 5A and B). To further investigate the regulated mechanism, promoter activity experiments were conducted to examine whether KLF9 directly bound to the DNA element of $C O X-2$. The promoter region of $C O X-2$ was fused to a luciferase reporter gene and was co-transfected with KLF9 expression plasmid into RAW264.7 cells. Notably, KLF9 significantly inhibited $C O X-2$ promoter activity in RAW264.7 cells under LPS-inducing conditions. 
A
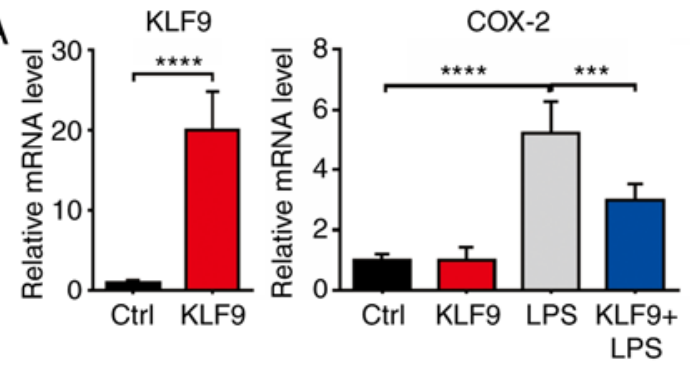

B

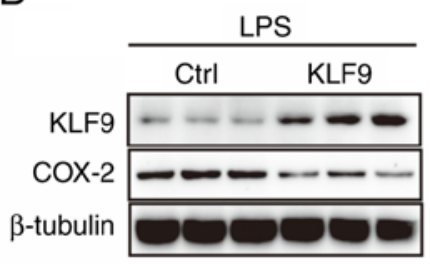

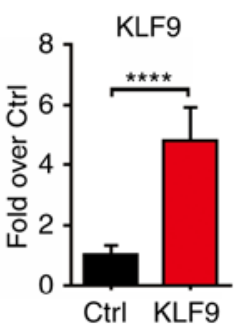

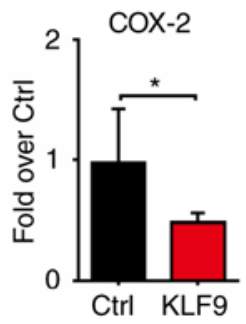

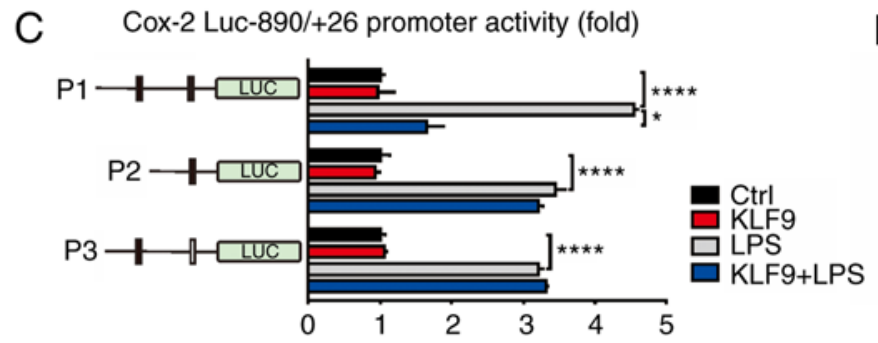
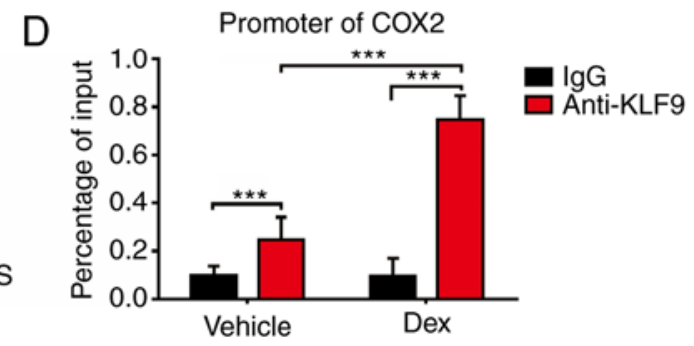

Figure 5. KLF9 suppresses LPS-induced COX-2 expression. (A and B) Real-time quantitative PCR and western blot analysis revealed the expression levels of COX-2 with KLF9 overexpression and control RAW264.7 cells following treatment with $1 \mu \mathrm{g} / \mathrm{ml}$ LPS or saline. (C) The promoter region of COX-2 was cloned and fused to a luciferase reporter system. The deleted and mutated promoters were co-transfected into RAW264.7 cells along with pcDNA3.1 or KLF9 expression plasmid. Luciferase activity was corrected for Renilla luciferase activity and normalized to control groups. (D) ChIP analysis in RAW264.7 cells following LPS treatment. Experiments were performed in triplicate. Error bars represent the standard deviation $\left({ }^{*} \mathrm{P}<0.05 ;{ }^{* * * *} \mathrm{P}<0.005 ;{ }^{* * * * *} \mathrm{P}<0.001\right)$. KLF9, Krüppel-like factor 9; COX-2, cyclooxygenase-2; ChIP, chromatin immunoprecipitation; LPS, lipopolysaccharide.

To determine the region responsible for KLF9 suppression, a series of deletions of $C O X-2$ promoter was constructed. As revealed, KLF9 significantly suppressed the P1 promoter activity, whereas the suppression was almost completely abolished with the transfection of $\mathrm{P} 2$ and mutant promoters under LPS-inducing conditions (Fig. 5C). ChIP assays using RAW264.7 cell extracts indicated that KLF9 proteins were recruited to the $C O X-2$ promoter, and this regulation was enhanced during Dex treatment (Fig. 5D). These data revealed the functional involvement of KLF9 in the regulation of $C O X-2$ gene transcription.

\section{Discussion}

GCs have been used for the early treatment of rheumatoid arthritis, and have since become the most common therapy for inflammatory disorders (4). GCs remain very effective anti-inflammatory and immunosuppressive agents used in the treatment of numerous autoimmune diseases (1). However, recent research has suggested that chronic use of GCs is associated with tumorigenesis. Although various modes of action are still debated, it is broadly accepted that GCs mediate their anti-inflammatory and immunosuppressive effects by affecting macrophage viability and antitumor functionality (4). Several studies have demonstrated that tumor-derived KLF9 may act as a tumor suppressor by promoting apoptosis $(8,19,20)$. Decreased KLF9 suppresses oxidative stress and apoptosis, subsequently promoting cancer progression (7). In fact, KLF9 overexpression in tumor cells can induce apoptosis via excessive oxidative stress production. However, whether macrophage-derived KLF9 exerts the same effect is yet to be elucidated. Previous research has reported that Dex promotes macrophage apoptosis and decreases Cyt- $c$, caspase-3, and caspase-9 expression (21). Research has demonstrated that
Dex promoted macrophage apoptosis partly by affecting the mitochondrial apoptosis pathway. The present findings indicated that Dex induced GR recruitment to the KLF9 promoter, consequently increasing the levels of KLF9, and increasing mitochondrial ROS production, leading to mitochondrial-dependent apoptosis of macrophages. Therefore, a molecular mechanism indicating KLF9 as an immunosuppressor and an important regulator of GCs-induced macrophage apoptosis is proposed. In the present study, it was demonstrated that decreased KLF9 levels could alleviate Dex-induced macrophage apoptosis. Altered levels of caspase-3, caspase-9, and Cyt- $c$ following overexpression of KLF9 in accordance with exposure to Dex were also revealed. In addition, it has been reported that KLF9 causes intracellular ROS accumulation by suppressing transcription of the thioredoxin reductase 2 gene, which plays a pivotal role in defense against oxidative damage $(7,19)$. In the present study, it was revealed that KLF9 could promote the accumulation of intracellular ROS, thus inducing macrophage apoptosis. The present data indicated a hormone-responsive model of KLF9-dependent apoptosis regulation through the mitochondrial pathway.

The increased understanding regarding GCs regulation of tumor progression has drawn attention to the therapeutic potential of modulating apoptosis and the antitumor functions of macrophages. Suppression of inflammatory factors benefits tumor growth via KLF9 overexpression in macrophages. The contribution of tumor-derived COX-2 to tumorigenesis has been examined in numerous studies (14,22-24). COX-2 is associated with a poor prognosis across a range of human cancers $(13,25)$. In the present study, it was revealed that KLF9 suppressed macrophage-derived COX-2 levels. COX-2 and numerous inflammatory factors are induced during stimulation of macrophages by LPS (24). This suggests that elevated levels of macrophage-derived COX-2 is strongly 
related to antitumor effects (14). While this implies that the microenvironment may change, which would support tumor growth due to prostaglandin production by COX-2 enzymatic activity, other mechanisms are possible. Decreased levels of COX-2 are relative to the abnormal immune function of macrophages $(17,18)$. In the present study, it was revealed that KLF9, a direct target of GCs, could reduce LPS-induced COX-2 levels, indicating that KLF9 is a potent inhibitor of immune function in macrophages.

Constitutive downregulation of KLF9 has been revealed in many types of cancer. However, it was revealed that KLF9 induction by GCs in macrophages, originally administered for anti-inflammatory purposes, conferred the risk of tumorigenesis. Furthermore, the molecular mechanism of the aforementioned effect revealed that KLF9 is an important responder to small molecule hormones and modulates oxidative injury and cell death. GR acts as an upstream regulator to stimulate expression of $K L F 9$. It was also revealed that KLF9 directly bound to the COX-2 promoter, and moderately suppressed the COX-2 transcriptional activity in a luciferase reporter assay, indicating potential contribution to $\mathrm{COX}-2$ inactivation.

Collectively, these observations indicate that KLF9 mediates macrophage apoptosis by directly activating the mitochondrial pathway. Most studies regarding KLF9 in cancer have focused on global inhibition of KLF9, especially in the tumor cells themselves, as a therapeutic target. Despite consensus that interruption of KLF9 function in cancer cells reduces tumorigenesis in in vivo models, an established macrophage hazard associated with selectively suppressed KLF9 levels severely limits their clinical use $(26,27)$. This hazard arises, since in addition to the desired inhibition of KLF9 in the injured tissue, unwanted collateral augmentation of macrophage KLF9 levels reduces the inflammatory response (11). By avoiding increased levels of macrophage KLF9, specific small molecule drugs targeting KLF9 may reduce the antitumor effect, while providing the desired antitumor outcome. To the best of our knowledge, the present finding are the first to specifically investigate KLF9 levels in macrophages in relation to tumorigenesis.

In summary, it is proposed that two functional changes of GCs inducing KLF9 upregulation in macrophages may contribute to this antitumor shift. Firstly, KLF9 induced macrophage apoptosis through the mitochondrial-dependent pathway. Increased KLF9 levels disrupted the mitochondrial membrane potential and increased ROS, subsequently promoting the release of $\mathrm{Cyt}-c$, which is pivotal for the mitochondrial-dependent apoptosis pathway. Conversely, KLF9 suppression restored the membrane potential during macrophage exposure to Dex. KLF9 knockdown reversed Dex-induced cleaved caspase-3 level in macrophages. These results indicated that KLF9 participated in Dex-induced macrophage apoptosis through the mitochondrial-dependent apoptosis pathway. Furthermore, it was revealed that specific inhibition of COX-2 by KLF9 in macrophages was administered for immunosuppression purposes incidentally increasing the likelihood of cancer development. Since increased KLF9 levels promote oxidative stress, downregulation may promote the immune function of macrophages. Thus, it is important to evaluate the therapeutic value of KLF9 in macrophages.
Moreover, as an important target of GCs, dual targeting of KLF9 could be promising in both oxidative injury and cancer treatment.

\section{Acknowledgements}

Not applicable.

\section{Funding}

No funding was received.

\section{Availability of data and materials}

The datasets used during the present study are available from the corresponding author upon reasonable request.

\section{Authors' contributions}

JL analyzed and interpreted the data and wrote the manuscript. FA performed most of the experiments. GZ, WL and BL assisted in the completion of the experiments. All authors read and approved the final manuscript and agree to be accountable for all aspects of the research in ensuring that the accuracy or integrity of any part of the work are appropriately investigated and resolved.

\section{Ethics approval and consent to participate}

Not applicable.

\section{Patient consent for publication}

Not applicable.

\section{Competing interests}

The authors declare that they have no competing interests.

\section{References}

1. Cari L,De Rosa F,Nocentini G and Riccardi C: Context-dependent effect of glucocorticoids on the proliferation, differentiation, and apoptosis of regulatory $\mathrm{T}$ cells: A review of the empirical evidence and clinical applications. Int J Mol Sci 20: pii: E1142, 2019.

2. Mylka V, Deckers J, Ratman D, De Cauwer L, Thommis J, De Rycke R,Impens F, LibertC, Tavernier J, Vanden Berghe W, et al: The autophagy receptor SQSTM1/p62 mediates anti-inflammatory actions of the selective $\mathrm{NR} 3 \mathrm{C} 1 /$ glucocorticoid receptor modulator compound A (CpdA) in macrophages. Autophagy 14: 2049-2064, 2018.

3. Patil RH, Naveen Kumar M, Kiran Kumar KM, Nagesh R, Kavya K, Babu RL, Ramesh GT and Chidananda Sharma S: Dexamethasone inhibits inflammatory response via down regulation of AP-1 transcription factor in human lung epithelial cells. Gene 645: 85-94, 2018.

4. Achuthan A, Aslam ASM, Nguyen Q, Lam PY, Fleetwood AJ, Frye AT, Louis C, Lee MC, Smith JE, Cook AD, et al: Glucocorticoids promote apoptosis of proinflammatory monocytes by inhibiting ERK activity. Cell Death Dis 9: 267, 2018.

5. Li B, Wang Y, Yin L, Huang G, Xu Y, Su J, Ma L and Lu J: Glucocorticoids promote the development of azoxymethane and dextran sulfate sodium-induced colorectal carcinoma in mice. BMC Cancer 19: 94, 2019. 
6. Wang C, Nanni L, Novakovic B, Megchelenbrink W, Kuznetsova T, Stunnenberg HG, Ceri S and Logie C: Extensive epigenomic integration of the glucocorticoid response in primary human monocytes and in vitro derived macrophages. Sci Rep 9: $2772,2019$.

7. Bagati A, Moparthy S, Fink EE, Bianchi-Smiraglia A, Yun DH, Kolesnikova M, Udartseva OO, Wolff DW, Roll MV, Lipchick BC, et al: KLF9-dependent ROS regulate melanoma progression in stage-specific manner. Oncogene 38: 3585-3597, 2019.

8. Zhong Z, Zhou F, Wang D, Wu M, Zhou W, Zou Y, Li J, Wu L and Yin X: Expression of KLF9 in pancreatic cancer and its effects on the invasion, migration, apoptosis, cell cycle distribution, and proliferation of pancreatic cancer cell lines. Oncol Rep 40: 3852-3860, 2018

9. Fink EE, Moparthy S, Bagati A, Bianchi-Smiraglia A, Lipchick BC, Wolff DW, Roll MV, Wang J, Liu S, Bakin AV, et al: XBP1-KLF9 axis acts as a molecular rheostat to control the transition from adaptive to cytotoxic unfolded protein response. Cell Rep 25: 212-223 e4, 2018.

10. Glinghammar B, Skogsberg J, Hamsten A and Ehrenborg E: PPARdelta activation induces COX-2 gene expression and cell proliferation in human hepatocellular carcinoma cells. Biochem Biophys Res Commun 308: 361-368, 2003.

11. Barnard ME, Hecht JL, Rice MS, Gupta M, Harris HR, Eliassen AH, Rosner BA, Terry KL and Tworoger SS: Anti-inflammatory drug use and ovarian cancer risk by COX1/COX2 expression and infiltration of tumor-associated macrophages. Cancer Epidemiol Biomarkers Prev 27: 1509-1517, 2018.

12. Lanocha-Arendarczyk N, Baranowska-Bosiacka I, Kot K, Gutowska I, Kolasa-Wołosiuk A, Chlubek D and Kosik-Bogacka D: Expression and activity of COX-1 and COX-2 in Acanthamoeba sp.-Infected lungs according to the host immunological status. Int J Mol Sci 19: pii: E121, 2018.

13. Kern MA, Haugg AM, Koch AF, Schilling T, Breuhahn K, Walczak H, Fleischer B, Trautwein C, Michalski C, Schulze-Bergkamen $\mathrm{H}$, et al: Cyclooxygenase-2 inhibition induces apoptosis signaling via death receptors and mitochondria in hepatocellular carcinoma. Cancer Res 66: 7059-7066, 2006.

14. Chen EP, Markosyan N, Connolly E, Lawson JA, Li X, Grant GR, Grosser T, FitzGerald GA and Smyth EM: Myeloid Cell COX-2 deletion reduces mammary tumor growth through enhanced cytotoxic T-lymphocyte function. Carcinogenesis 35: 1788-1797, 2014.

15. Chen EP and Smyth EM: COX-2 and PGE2-dependent immunomodulation in breast cancer. Prostaglandins Other Lipid Mediat 96: 14-20, 2011.

16. Carey LC, Valego NK, Chen K and Rose JC: Thyroid hormone regulates renocortical COX-2 and PGE2 expression in the late gestation fetal sheep. Reprod Sci 15: 598-603, 2008

17. Zhang Y, Zhou Y, Chen S, Hu Y, Zhu Z, Wang Y, Du N, Song T, Yang Y, Guo A and Wang Y: Macrophage migration inhibitory factor facilitates prostaglandin E2 production of astrocytes to tune inflammatory milieu following spinal cord injury. J Neuroinflammation 16: 85, 2019.
18. Hwang JH, Ma JN, Park JH, Jung HW and Park YK: Anti-inflammatory and antioxidant effects of MOK, a polyherbal extract, on lipopolysaccharidestimulated RAW 264.7 macrophages. Int J Mol Med 43: 26-36, 2019.

19. Yang D, Lv Z, Zhang H, Liu B, Jiang H, Tan X, Lu J, Baiyun R and Zhang Z: Activation of the Nrf2 signaling pathway involving KLF9 plays a critical role in allicin resisting against arsenic trioxide-induced hepatotoxicity in rats. Biol Trace Elem Res 176: 192-200, 2017.

20. Bagheri-Yarmand R, Sinha KM, Li L, Lu Y, Cote GJ, Sherman SI and Gagel RF: Combinations of tyrosine kinase inhibitor and ERAD inhibitor promote oxidative stress-induced apoptosis through ATF4 and KLF9 in medullary thyroid cancer. Mol Cancer Res 17: 751-760, 2019.

21. Yang Y, Wei H, Song T, Cai A, Zhou Y, Peng J, Jiang S and Peng J: E4BP4 mediates glucocorticoid-regulated adipogenesis through COX2. Mol Cell Endocrinol 450: 43-53, 2017.

22. Majumder M,Xin X,Liu L, Tutunea-Fatan E, Rodriguez-Torres M, Vincent K, Postovit LM, Hess D and Lala PK: COX-2 induces breast cancer stem cells via EP4/PI3K/AKT/NOTCH/WNT axis. Stem Cells 34: 2290-2305, 2016.

23. Lin HY, Davis PJ, Tang HY, Mousa SA, Luidens MK, Hercbergs AH and Davis FB: The pro-apoptotic action of stilbene-induced COX-2 in cancer cells: Convergence with the anti-apoptotic effect of thyroid hormone. Cell Cycle 8: 1877-1882, 2009.

24. Li Y, Zou L, Li T, Lai D, Wu Y and Qin S: Mogroside V inhibits LPS-induced COX-2 expression/ROS production and overexpression of HO-1 by blocking phosphorylation of AKT1 in RAW264.7 cells. Acta Biochim Biophys Sin (Shanghai) 51: 365-374, 2019

25. Chang J, Xue M, Yang S, Yao B, Zhang B, Chen X, Pozzi A and Zhang MZ: Inhibition of $11 \beta$-Hydroxysteroid dehydrogenase Type II suppresses lung carcinogenesis by blocking tumor COX-2 expression as well as the ERK and mTOR signaling pathways. PLoS One 10: e0127030, 2015.

26. Park J, Ha SH, Abekura F, Lim H, Magae J, Ha KT, Chung TW, Chang YC,Lee YC,ChungE, etal:4-O-Carboxymethylascochlorin inhibits expression levels of on inflammation-related cytokines and matrix metalloproteinase-9 through NF- $\kappa \mathrm{B} / \mathrm{MAPK} / \mathrm{TLR} 4$ signaling pathway in LPS-activated RAW264.7 cells. Front Pharmacol 10: 304, 2019.

27. Greene ER, Huang S, Serhan CN and Panigrahy D: Regulation of inflammation in cancer by eicosanoids. Prostaglandins Other Lipid Mediat 96: 27-36, 2011.

This work is licensed under a Creative Commons Attribution-NonCommercial-NoDerivatives 4.0 International (CC BY-NC-ND 4.0) License. 